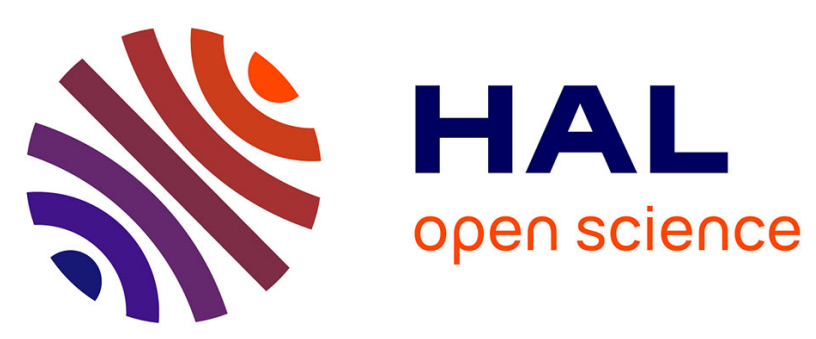

\title{
On the Capacity of Full-Duplex Causal Cognitive Interference Channels to within a Constant Gap
}

Martina Cardone, Daniela Tuninetti, Raymond Knopp, Umer Salim

\section{To cite this version:}

Martina Cardone, Daniela Tuninetti, Raymond Knopp, Umer Salim. On the Capacity of Full-Duplex Causal Cognitive Interference Channels to within a Constant Gap. IEEE International Conference on Communications (ICC), 2014, Jun 2014, Australia. pp.1-5. hal-00981886

\section{HAL Id: hal-00981886 https://hal.science/hal-00981886}

Submitted on 23 Jul 2014

HAL is a multi-disciplinary open access archive for the deposit and dissemination of scientific research documents, whether they are published or not. The documents may come from teaching and research institutions in France or abroad, or from public or private research centers.
L'archive ouverte pluridisciplinaire HAL, est destinée au dépôt et à la diffusion de documents scientifiques de niveau recherche, publiés ou non, émanant des établissements d'enseignement et de recherche français ou étrangers, des laboratoires publics ou privés. 


\title{
On the Capacity of Full-Duplex Causal Cognitive Interference Channels to within a Constant Gap
}

\author{
Martina Cardone ${ }^{\dagger}$, Daniela Tuninetti*, Raymond Knopp ${ }^{\dagger}$, Umer Salim $^{\ddagger}$ \\ ${ }^{\dagger}$ Eurecom, Biot, 06410, France, Email: \{cardone, knopp\}@eurecom.fr \\ * University of Illinois at Chicago, Chicago, IL 60607, USA, Email: danielat@uic.edu \\ $\ddagger$ Intel Mobile Communications, Sophia Antipolis, 06560, France, Email: umer.salim@intel.com
}

\begin{abstract}
This paper considers the two-user Gaussian Causal Cognitive Interference Channel (GCCIC), which consists of two source-destination pairs that share the same channel and where one full-duplex cognitive source can causally learn the message of the primary source through a noisy link. The GCCIC is an interference channel with unilateral source cooperation that models practical cognitive radio networks. Different achievable strategies are shown to be at most a finite number of bits away from an outer bound for a set of the channel parameters that, roughly speaking, excludes the case of weak interference at both receivers.
\end{abstract}

\section{INTRODUCTION}

We examine the system in Fig. 1, consisting of two sourcedestination pairs sharing the same channel. One cognitive source, indicated as CTx, overhears the other primary source, indicated as PTx, through a noisy channel. The CTx, then, in addition to sending its own data, cooperates with the PTx in sending the data to the primary receiver, PRx. This system is an interference channel with unilateral source cooperation that models a practical cognitive radio overlay technology [1]. The prime features of this model are to firstly allow the cognitive nodes to obtain spectral resources for their communication without hindering the communication of the primary nodes, and secondly to maintain or enhance the communication performance of the primary nodes. Contrary to the commonly studied cognitive radio model that assumes a perfect noncausal primary message knowledge at the CTx [2], in this work we treat the causal case, i.e., the CTx has access only to primary information it receives over the air. We refer to this system as the Causal Cognitive Interference Channel (CCIC).

The CCIC fits future 4G networks with heterogeneous deployments [3] where the CTx corresponds to the so-called small-cell base-station, or eNB. In this scenario, CTx would listen to PTx transmission but not make use of a dedicated point-to-point backhaul link. We consider deployment scenarios where the $\mathrm{CTx} \rightarrow \mathrm{CRx}$ link is on the same carrier frequency as PTx $\rightarrow$ PRx link and the CTx works in full-duplex mode.

Related Work: The presence of a lossy communication link between PTx and CTx enables CTx to cooperate with PTx. CTx, in fact, through this noisy channel overhears PTx's transmissions and gathers information about PTx's message. This serves as the basis for unilateral cooperation, which is a special case of the IC with generalized feedback, or bilateral source cooperation, which has received considerable attention lately. Several outer bounds have been developed for the IC

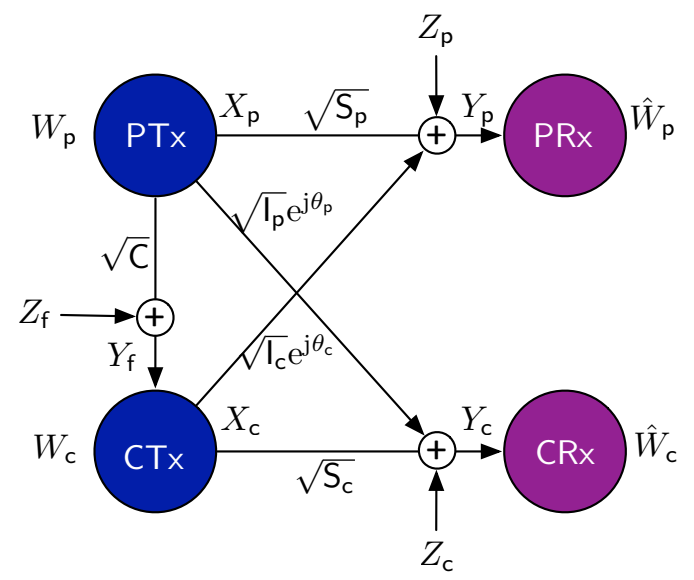

Fig. 1: The Gaussian causal cognitive interference channel.

with bilateral source cooperation [4], [5], [6] and a number of achievable schemes have been proposed as well [7]. The achievable region in $[7, \mathrm{Sec} . \mathrm{V}]$ is, to the best of our knowledge, the largest known; the strategy exploits partial-decodeand-forward relaying, superposition coding, rate splitting and Gelfand-Pinsker binning (or Dirty Paper Coding (DPC) [8] in the Gaussian noise case). For the two-user Gaussian noise IC with bilateral source cooperation, under the assumption of equally strong cooperation links, the scheme of $[7, \mathrm{Sec} . \mathrm{V}]$ was sufficient to match the sum-capacity upper bounds of [6], [5] to within a constant gap [5], [9]. In particular, [5] characterized the sum-capacity to within 10 bits/user of the IC with bilateral source cooperation with arbitrary direct and interfering links. The gap was reduced to 2 bits/user in the "strong cooperation regime' in [9] with symmetric direct and interfering links. The IC with unilateral source cooperation, which is a special case of bilateral cooperation where the cooperation capabilities are not restricted to be the same, represents a more realistic scenario for cognitive radio networks.

The cognitive radio channel is usually modeled following the work of Devroye et al. [2], in which the superior capabilities of the CTx are modeled as perfect non-causal knowledge of PTx's message at CTx. For this non-causal model the capacity region in Gaussian noise is known exactly for some parameter regimes and to within 1 bit otherwise [10]. In this work we remove the ideal non-causal message knowledge 
assumption by considering a more realistic scenario where CTx causally learns the PTx's message through a noisy link. The IC with unilateral source cooperation was studied in [11], where it was assumed that at any given time instant the CTx has a non-causal access to $L \geq 0$ future channel outputs. The case $L=0$ corresponds to the strictly causal case considered in this paper. The authors of [11] derived potentially tighter outer bounds for the CCIC than those of [5], [6] specialized to unilateral source cooperation; however it is not clear how to evaluate these bounds in Gaussian noise since they are expressed as a function of auxiliary random variables jointly distributed with the inputs and for which no cardinality bounds on the corresponding alphabets are known. As remarked in [11, Rem. 2, point 6], the achievable region in [11, Cor. 1] is also no smaller than the one in [7, Sec. V] specialized to unilateral source cooperation. Although [11, Cor. 1] is, to the best of our knowledge, the largest known achievable region for the general memoryless CCIC, its evaluation in general is not simple as it comprises 9 jointly distributed auxiliary random variables and 30 rate constraints. In [11] it was noted that, for some simulated sets of channel gains, the proposed outer and inner bounds are not far away from one another. However, a general performance guarantee in terms of capacity to within a constant gap was not given.

Contributions and Paper Organization: The rest of the paper is organized as follows. Section II describes the channel model and summarizes known outer bounds. Section III characterizes the capacity region of the GCCIC to within 2 bits/user for a large set of channel parameters that, roughly speaking, excludes the case of weak interference at both receivers. This is, to the best of our knowledge, the first result on the capacity (and not only sum-capacity) guarantee for an IC with source cooperation. Moreover, to the best of our knowledge, the case of asymmetric cooperation links, of which unilateral cooperation is a special case, has not been considered in the literature. Section IV concludes the paper. The derivation of the achievable scheme is in the Appendix.

\section{The GAUSSIAN NOISE CHANNEL}

We adopt the following notation: $[x]^{+}:=\max \{0, x\}$ for $x \in \mathbb{R} ; \log ^{+}(x):=\max \{0, \log (x)\}$ for $x \in \mathbb{R}^{+}$; the subscript c, resp. p, is used for quantities related to the cognitive pair, resp. primary pair. The subscript $f$ is used to refer to generalized feedback information received at the CTx.

A CCIC has two transmitters, PTx and CTx. Each source has a message that has to be reliably decoded at the corresponding receiver. These messages are sent through a shared memoryless channel. We use the standard definition of capacity, which we do not repeat for sake of space.

A single-antenna full-duplex GCCIC, shown in Fig. 1, is described by the input/output relationship

$$
\left[\begin{array}{c}
Y_{\mathrm{f}} \\
Y_{\mathrm{p}} \\
Y_{\mathrm{c}}
\end{array}\right]=\left[\begin{array}{cc}
\sqrt{\mathrm{C}} & \star \\
\sqrt{\mathrm{S}_{\mathrm{p}}} & \sqrt{I_{\mathrm{c}}} \mathrm{e}^{\mathrm{j} \theta_{\mathrm{c}}} \\
\sqrt{I_{\mathrm{p}}} \mathrm{e}^{\mathrm{j} \theta_{\mathrm{p}}} & \sqrt{\mathrm{S}_{\mathrm{c}}}
\end{array}\right]\left[\begin{array}{c}
X_{\mathrm{p}} \\
X_{\mathrm{c}}
\end{array}\right]+\left[\begin{array}{c}
Z_{\mathrm{f}} \\
Z_{\mathrm{p}} \\
Z_{\mathrm{c}}
\end{array}\right],
$$

where $\star$ indicates the channel gain that does not affect the capacity region (because CTx can remove its transmit signal $X_{\mathrm{c}}$ from its channel output $Y_{\mathrm{f}}$ ). The channel gains are constant, and therefore known to all nodes. Without loss of generality certain channel gains can be taken to be real-valued and nonnegative because a node can compensate for the phase of one of its channel gains. The channel inputs are subject to a unitary power constraint, i.e., $\mathbb{E}\left[\left|X_{i}\right|^{2}\right] \leq 1, i \in\{\mathrm{p}, \mathrm{c}\}$. This assumption is without loss of generality because nonunitary power constraints can be incorporated into the channel gains. The noises are independent Gaussian random variables with, without loss of generality, zero mean and unit variance. Notice that, the classical noncooperative IC is obtained as a special case of the CCIC by setting $\mathrm{C}=0$ and the non-causal cognitive IC in the limit for $\mathrm{C} \rightarrow+\infty$.

The capacity of the channel in (1) is unknown. The capacity region of the GCCIC is said to be known to within GAP bits/user if we can show an inner bound region $\mathcal{I}$ and an outer bound region $\mathcal{O}$ such that $\left(R_{\mathrm{p}}, R_{\mathrm{c}}\right) \in \mathcal{O} \Longrightarrow$ $\left(\left[R_{\mathrm{p}}-\mathrm{GAP}\right]^{+},\left[R_{\mathrm{c}}-\mathrm{GAP}\right]^{+}\right) \in \mathcal{I}$, where $R_{\mathrm{p}} \in \mathbb{R}^{+}$, resp. $R_{\mathrm{c}} \in \mathbb{R}^{+}$, is the transmission rate (in bits per channel use) for PTx, resp. CTx. We remark that [5], [9] characterized, to within a constant gap, the sum-capacity of the IC with bilateral source cooperation under the assumption of equally strong cooperation links. To the best of our knowledge, the case of asymmetric cooperation links, of which unilateral cooperation is a special case, has not been considered in the literature. Moreover, to the best of our knowledge, the whole capacity region with source cooperation has never been characterized to within a constant gap in the literature, which is a major contribution of this work.

Known Outer Bounds: In the literature several outer bounds are known for bilateral source cooperation [4], [5], [6]. Here we specialize some of them for the GCCIC in (1) so as to obtain

$$
\begin{aligned}
R_{\mathrm{c}} & \leq \log \left(1+\mathrm{S}_{\mathrm{c}}\right) \\
R_{\mathrm{p}} & \leq \log \left(1+\left(\sqrt{\mathrm{S}_{\mathrm{p}}}+\sqrt{\mathrm{I}_{\mathrm{c}}}\right)^{2}\right), \\
R_{\mathrm{p}} & \leq \log \left(1+\mathrm{C}+\mathrm{S}_{\mathrm{p}}\right) \\
R_{\mathrm{p}}+R_{\mathrm{c}} \leq & \log ^{+}\left(\frac{1+\mathrm{S}_{\mathrm{c}}}{1+\mathrm{I}_{\mathrm{c}}}\right)+\log \left(1+\left(\sqrt{\mathrm{S}_{\mathrm{p}}}+\sqrt{\mathrm{I}_{\mathrm{c}}}\right)^{2}\right), \\
R_{\mathrm{p}}+R_{\mathrm{c}} \leq & \log \left(\frac{1+\mathrm{C}+\max \left\{\mathrm{S}_{\mathrm{p}}, \mathrm{I}_{\mathrm{p}}\right\}}{1+\mathrm{I}_{\mathrm{p}}}\right) \\
& +\log \left(1+\left(\sqrt{\mathrm{S}_{\mathrm{c}}}+\sqrt{\mathrm{I}_{\mathrm{p}}}\right)^{2}\right)
\end{aligned}
$$

where the bounds on the individual rates are cut-set bounds, and the sum-rate bounds are from [6]. More details can be found in the journal version of this paper [12].

The upper bound in (2) for $C \rightarrow+\infty$ reduces to the upper bound for the non-causal cognitive IC in [10, Th. III.1], which unifies previously known outer bounds for the weak $\left(\mathrm{S}_{\mathrm{c}}>\mathrm{I}_{\mathrm{c}}\right)$ and strong $\left(S_{c} \leq I_{c}\right)$ interference regimes. The region in [10, Th. III.1] is known to be achievable to within 1 bit/user in all parameter regimes. In weak interference $\left(S_{c}>I_{c}\right)$, the capacity 
region of the non-causal cognitive IC is known exactly and is given by

$$
\begin{aligned}
& R_{\mathrm{p}} \leq \log \left(1+\frac{\mathrm{S}_{\mathrm{p}}+\left|\gamma_{\mathrm{c}}\right|^{2} \mathrm{I}_{\mathrm{c}}+2\left|\gamma_{\mathrm{c}}\right| \sqrt{\mathrm{S}_{\mathrm{p}} \mathrm{I}_{\mathrm{c}}}}{1+\left(1-\left|\gamma_{\mathrm{c}}\right|^{2}\right) \mathrm{I}_{\mathrm{c}}}\right), \\
& R_{\mathrm{c}} \leq\left(1+\left(1-\left|\gamma_{\mathrm{c}}\right|^{2}\right) \mathrm{S}_{\mathrm{c}}\right),
\end{aligned}
$$

union over all $\left|\gamma_{c}\right| \leq 1$. Therefore, the region in (3) is an outer bound for the GCCIC for $S_{c}>I_{c}$.

\section{THE CAPACITY REGION TO WITHIN A CONSTANT GAP}

In this section we prove that the outer bounds in (2) and (3) are achievable to within a constant gap for a large set of channel parameters that, roughly speaking, excludes the case of weak interference at both receivers.

Our main result for the general GCCIC is as follows:

Theorem 1. For the general GCCIC we have:

A) $\mathrm{C} \leq \mathrm{S}_{\mathrm{p}}, \mathrm{S}_{\mathrm{c}} \mathrm{S}_{\mathrm{p}} \leq\left(1+\mathrm{I}_{\mathrm{p}}\right)\left(1+\mathrm{I}_{\mathrm{c}}\right)$ : capacity to within 2 bits/user with a noncooperative scheme,

B) $\mathrm{S}_{\mathrm{p}}<\mathrm{C} \leq \mathrm{I}_{\mathrm{p}}$ : capacity to within 1 bit/user with a cooperative scheme based on superposition coding,

C) $\max \left\{\mathrm{S}_{\mathrm{p}}, \mathrm{I}_{\mathrm{p}}\right\}<\mathrm{C}, \mathrm{S}_{\mathrm{c}} \frac{1+\mathrm{I}_{\mathrm{p}}+\mathrm{S}_{\mathrm{p}}}{1+22 \mathrm{I}_{\mathrm{p}}} \leq \mathrm{I}_{\mathrm{c}}, \mathrm{S}_{\mathrm{c}} \leq \mathrm{I}_{\mathrm{c}}$ : capacity to within 1.8 bits/user with a cooperative scheme based on superposition coding,

D) $\mathrm{S}_{\mathrm{c}}>\mathrm{I}_{\mathrm{c}}$ and $\mathrm{C} \geq\left(\mathrm{S}_{\mathrm{p}}+\mathrm{I}_{\mathrm{c}}+2 \sqrt{\mathrm{S}_{\mathrm{p}} \mathrm{I}_{\mathrm{c}} \frac{\mathrm{I}_{\mathrm{p}}}{1+\mathrm{I}_{\mathrm{p}}}}\right)\left(1+\mathrm{I}_{\mathrm{p}}\right)$ : capacity to within 1 bit/user with a cooperative scheme based on DPC.

The rest of the section is devoted to the proof of Th. 1. We divide the whole set of parameters depending on the strength of the cooperation link $C$ compared to the direct link $S_{p}$ and the interference link $I_{p}$. We discuss each regime separately. Fig. 2 shows the regimes (except regime D) of Th. 1 for which we have an approximate capacity result.

\section{A. The case $\mathrm{C} \leq \mathrm{S}_{\mathrm{p}}$}

Under the condition $C \leq S_{p}$ the outer bound in (2) can be further bounded as

$$
\begin{aligned}
R_{\mathrm{c}} & \leq \log \left(1+\mathrm{S}_{\mathrm{c}}\right), \\
R_{\mathrm{p}} & \leq \log \left(1+\mathrm{S}_{\mathrm{p}}\right)+\log (2), \\
R_{\mathrm{p}}+R_{\mathrm{c}} & \leq \log ^{+}\left(\frac{1+\mathrm{S}_{\mathrm{c}}}{1+\mathrm{I}_{\mathrm{c}}}\right)+\log \left(1+\mathrm{S}_{\mathrm{p}}+\mathrm{I}_{\mathrm{c}}\right)+\log (2), \\
R_{\mathrm{p}}+R_{\mathrm{c}} & \leq \log ^{+}\left(\frac{1+\mathrm{S}_{\mathrm{p}}}{1+\mathrm{I}_{\mathrm{p}}}\right)+\log \left(1+\mathrm{S}_{\mathrm{c}}+\mathrm{I}_{\mathrm{p}}\right)+2 \log (2) .
\end{aligned}
$$

The bounds in (4) are to within 1 bit/user of

$$
\begin{aligned}
R_{\mathrm{c}} & \leq \log \left(1+\mathrm{S}_{\mathrm{c}}\right), \\
R_{\mathrm{p}} & \leq \log \left(1+\mathrm{S}_{\mathrm{p}}\right), \\
R_{\mathrm{p}}+R_{\mathrm{c}} & \leq \log \left(1+\mathrm{S}_{\mathrm{p}}+\mathrm{I}_{\mathrm{c}}\right)+\log ^{+}\left(\frac{1+\mathrm{S}_{\mathrm{c}}}{1+\mathrm{I}_{\mathrm{c}}}\right), \\
R_{\mathrm{p}}+R_{\mathrm{c}} & \leq \log \left(1+\mathrm{S}_{\mathrm{c}}+\mathrm{I}_{\mathrm{p}}\right)+\log ^{+}\left(\frac{1+\mathrm{S}_{\mathrm{p}}}{1+\mathrm{I}_{\mathrm{p}}}\right),
\end{aligned}
$$

which is a rate region achievable to within $1 \mathrm{bit} / \mathrm{user}$ for the noncooperative IC when the ' $R_{\mathrm{p}}+2 R_{\mathrm{c}}, 2 R_{\mathrm{p}}+R_{\mathrm{c}}$ '-type bounds in [13, Th. 3] are redundant; with the notation adopted in this paper, one can easily show that these bounds are redundant if

$$
\mathrm{S}_{\mathrm{c}} \mathrm{S}_{\mathrm{p}} \leq\left(1+\mathrm{I}_{\mathrm{p}}\right)\left(1+\mathrm{I}_{\mathrm{c}}\right) .
$$

Hence the noncooperative scheme of [13] is optimal to within 2 bits/user in the regime identified by (6) when the cooperation link gain satisfies $C \leq S_{p}$. Notice that the regime in (6), depicted in Fig. 2 (left), includes the strong interference regime and parts of the mixed and weak interference regimes.

Remark. When $S_{c} S_{p}>\left(1+I_{p}\right)\left(1+I_{c}\right)$ and $C \leq S_{p}$, in order to claim capacity to within a constant gap we must derive an upper bound that reduces to, or is to within a constant gap of, the capacity outer bound in [13, Th. 3] when $C=0$. The outer bound region in [13, Th. 3] is characterized by bounds on $R_{\mathrm{p}}+2 R_{\mathrm{c}} / 2 R_{\mathrm{p}}+R_{\mathrm{c}}$. Therefore, unless outer bounds on $2 R_{\mathrm{c}}+R_{\mathrm{p}}$ and $R_{\mathrm{c}}+2 R_{\mathrm{p}}$ for the cooperative case are developed, it is not possible to claim optimality to within a finite gap of the upper bound in (2) for small $\mathrm{C}$. The bounds were recently derived in [14]. In [15], the authors interpret the bounds on $2 R_{\mathrm{c}}+R_{\mathrm{p}}$ and $R_{\mathrm{c}}+2 R_{\mathrm{p}}$ as a measure of the amount of 'resource holes', or inefficiency, due to the distributed nature of the noncooperative IC. In [15], the authors showed that with output feedback from a destination to the intended source such 'resource holes' are no longer present, i.e., feedback enables coordination among the sources which results in a full utilization of the channel resources. An interesting open question is whether unilateral cooperation enables sufficient coordination among the sources for full utilization of the channel resources. In the limiting case where unilateral cooperation equals non-causal cognition, we know from [10] that the capacity region does not have bounds on $2 R_{\mathrm{c}}+R_{\mathrm{p}}$ and $R_{\mathrm{c}}+2 R_{\mathrm{p}}$, i.e., there are no 'resource holes'. The recent work in [14] partially answers the question in the regimes not covered in this paper.

B. The case $\mathrm{S}_{\mathrm{p}}<\mathrm{C} \leq \mathrm{I}_{\mathrm{p}}$

For $S_{p}<C \leq I_{p}$ we further bound the outer bound in (2) as

$$
\begin{aligned}
R_{\mathrm{c}} & \leq \log \left(1+\mathrm{S}_{\mathrm{c}}\right), \\
R_{\mathrm{p}} & \leq \log (1+\mathrm{C})+\log (2), \\
R_{\mathrm{p}}+R_{\mathrm{c}} & \leq \log ^{+}\left(\frac{1+\mathrm{S}_{\mathrm{c}}}{1+\mathrm{I}_{\mathrm{c}}}\right)+\log \left(1+\mathrm{S}_{\mathrm{p}}+\mathrm{I}_{\mathrm{c}}\right)+\log (2), \\
R_{\mathrm{p}}+R_{\mathrm{c}} & \leq \log \left(1+\mathrm{S}_{\mathrm{c}}+\mathrm{I}_{\mathrm{p}}\right)+2 \log (2) .
\end{aligned}
$$

The channel conditions suggest that PTx should take advantage of the strong cooperation link and send its message with the help of the CTx since $C>S_{p}$. The sum-rate upper bound in (7d) suggests that CRx should decode PTx's message in addition to its intended message, i.e., PTx should use a (cooperative) common message only. The sum-rate upper bound in (7c), suggests that PRx should decode CTx's message only when $I_{c}>S_{c}$, i.e., CTx should use both a (noncooperative) common and a (noncooperative) private message. This is exactly the strategy described in the Appendix with $Q=S_{1}=Z_{1}=\emptyset$, i.e., only a (cooperative) common message for the PTx carried by $V_{1}$. We apply Fourier-Motzkin elimination on the achievable region in (14) and we choose $V_{1}, U_{2}, T_{2}$ 


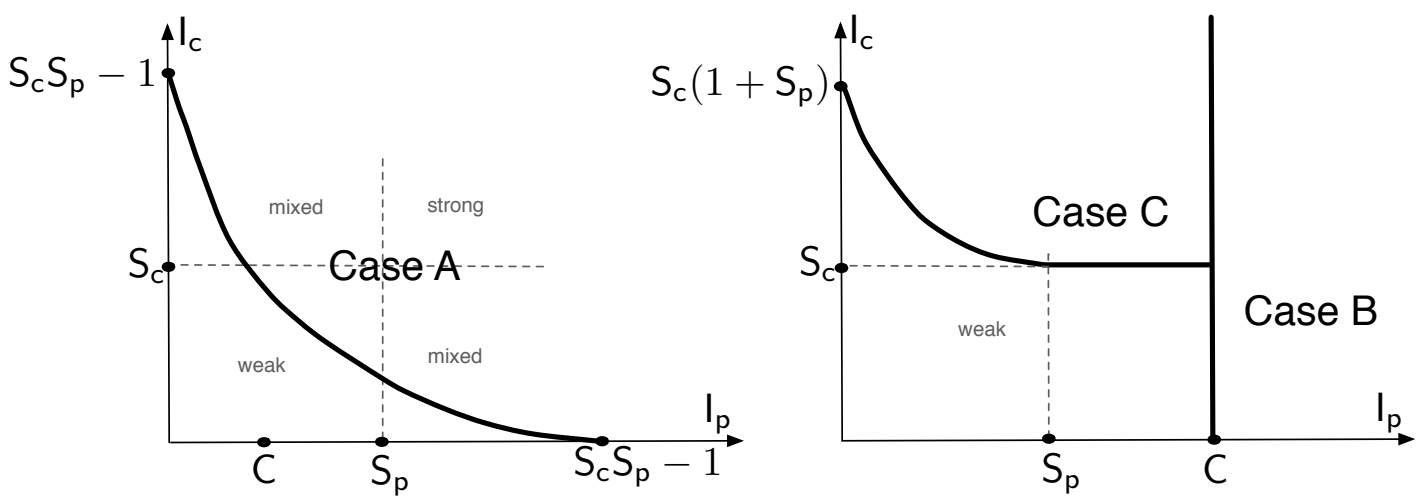

Fig. 2: The regimes identified by Theorem 1 .

to be i.i.d. $\mathcal{N}(0,1)$, and $X_{\mathrm{p}}=V_{1}, X_{\mathrm{c}}=\gamma_{\mathrm{c}} U_{2}+\sqrt{1-\left|\gamma_{\mathrm{c}}\right|^{2}} T_{2}$ with $\left|\gamma_{c}\right|=1$ if $S_{c} \leq I_{c}$ and $\left|\gamma_{c}\right|=0$ otherwise. With these choices we obtain that the following rate region is achievable

$$
\begin{aligned}
R_{\mathrm{c}} & \leq \log \left(1+\mathrm{S}_{\mathrm{c}}\right), \\
R_{\mathrm{p}} & \leq \log (1+\mathrm{C}), \\
R_{\mathrm{p}}+R_{\mathrm{c}} & \leq \log \left(1+\mathrm{S}_{\mathrm{p}}+\mathrm{I}_{\mathrm{c}}\right)+\log ^{+}\left(\frac{1+\mathrm{S}_{\mathrm{c}}}{1+\mathrm{I}_{\mathrm{c}}}\right), \\
R_{\mathrm{p}}+R_{\mathrm{c}} & \leq \log \left(1+\mathrm{S}_{\mathrm{c}}+\mathrm{I}_{\mathrm{p}}\right) .
\end{aligned}
$$

Notice that, although derived from a scheme based on DPC (see the Appendix for the details), the rate region in (8) is achieved with only superposition coding since $S_{1}=\emptyset$. By comparing the upper bound in (7) with the achievable region in (8) we conclude that the capacity region is known to within $1 \mathrm{bit} / \mathrm{user}$ for a general GCCIC where the channel gains satisfy $\mathrm{S}_{\mathrm{p}}<\mathrm{C} \leq \mathrm{I}_{\mathrm{p}}$. Notice that we did not impose any condition on the strength of $I_{c}$ compared to $S_{c}$, i.e., the result holds regardless of whether the interference at PRx is strong $\left(I_{c} \geq\right.$ $\mathrm{S}_{\mathrm{c}}$ ) or weak $\left(\mathrm{I}_{\mathrm{c}}<\mathrm{S}_{\mathrm{c}}\right)$ - see Fig. 2 'Case B' on the right.

C. The case $\mathrm{S}_{\mathrm{p}}<\mathrm{C}, \mathrm{I}_{\mathrm{p}}<\mathrm{C}$ and $\mathrm{S}_{\mathrm{c}} \leq \mathrm{I}_{\mathrm{c}}$

In this regime we further bound the outer bound in (2) as

$$
\begin{aligned}
R_{\mathrm{c}} & \leq \log \left(1+\mathrm{S}_{\mathrm{c}}\right), \\
R_{\mathrm{p}} & \leq \log (1+\mathrm{C})+\log (2), \\
R_{\mathrm{p}}+R_{\mathrm{c}} & \leq \log \left(1+\mathrm{S}_{\mathrm{p}}+\mathrm{I}_{\mathrm{c}}\right)+\log (2), \\
R_{\mathrm{p}}+R_{\mathrm{c}} & \leq \log \left(\frac{1+\mathrm{C}}{1+\mathrm{I}_{\mathrm{p}}}\right)+\log \left(1+\mathrm{S}_{\mathrm{c}}+\mathrm{I}_{\mathrm{p}}\right)+2 \log (2) .
\end{aligned}
$$

Here PTx takes advantage of the strong cooperation link, i.e., $\mathrm{C}>\mathrm{S}_{\mathrm{p}}$, and sends its message with the help of the CTx. The sum-rate upper bound in (9c) suggests that PRx should decode CTx's message in addition to its intended message, i.e., CTx should use a (noncooperative) common message only. The sum-rate upper bound in (9d) suggests that PTx should use both a (cooperative) common and a (cooperative) private message. This is exactly the strategy described in the Appendix with $Q=T_{2}=\emptyset$, i.e., only a (noncooperative) common message for CTx. Moreover, we do not bin $U_{2}$ against $S_{1}$, i.e., $I\left(S_{1} ; U_{2}\right)=0$. We apply Fourier-Motzkin elimination on the achievable region in (14) and we choose $V_{1}, S_{1}, Z_{1}, U_{2}$ to be i.i.d. $\mathcal{N}(0,1)$, and $X_{\mathrm{p}}=\sqrt{1-\left|\gamma_{\mathrm{p}}\right|^{2}} V_{1}+\gamma_{\mathrm{p}} Z_{1}, X_{\mathrm{c}}=$ $\gamma_{\mathrm{c}} S_{1}+\sqrt{1-\left|\gamma_{\mathrm{c}}\right|^{2}} U_{2}$ with the possible suboptimal choices $\left(1+I_{p}\right)\left|\gamma_{p}\right|^{2}=\left(1+S_{c}\right)\left|\gamma_{c}\right|^{2}=1$ inspired by [13]. With these choices we obtain that the following rate region is achievable

$$
\begin{aligned}
R_{\mathrm{c}} & \leq \log \left(1+\frac{\mathrm{S}_{\mathrm{c}} \frac{\mathrm{S}_{\mathrm{c}}}{1+\mathrm{S}_{\mathrm{c}}}}{1+\frac{\mathrm{I}_{\mathrm{p}}}{1+\mathrm{I}_{\mathrm{p}}}+\frac{\mathrm{S}_{\mathrm{c}}}{1+\mathrm{S}_{\mathrm{c}}}}\right), \\
R_{\mathrm{p}} & \leq \log (1+\mathrm{C}), \\
R_{\mathrm{p}}+R_{\mathrm{c}} & \leq \log \left(1+\mathrm{S}_{\mathrm{p}}+\mathrm{I}_{\mathrm{c}}\right), \\
R_{\mathrm{p}}+R_{\mathrm{c}} & \leq \log \left(1+\frac{\mathrm{C}}{1+\mathrm{I}_{\mathrm{p}}}\right)+\log (1+\Delta), \\
R_{\mathrm{p}}+R_{\mathrm{c}} & \leq \log \left(1+\frac{\mathrm{S}_{\mathrm{p}}}{1+\mathrm{I}_{\mathrm{p}}}+\frac{\mathrm{I}_{\mathrm{c}}}{1+\mathrm{S}_{\mathrm{c}}}\right)+\log (1+\Delta), \\
\Delta & :=\frac{\mathrm{S}_{\mathrm{c}} \frac{\mathrm{S}_{\mathrm{c}}}{1+\mathrm{S}_{\mathrm{c}}}+\mathrm{I}_{\mathrm{p}} \frac{\mathrm{I}_{\mathrm{p}}}{1+\mathrm{I}_{\mathrm{p}}}}{1+\frac{\mathrm{I}_{\mathrm{p}}}{1+\mathrm{I}_{\mathrm{p}}}+\frac{\mathrm{S}_{\mathrm{c}}}{1+\mathrm{S}_{\mathrm{c}}}} .
\end{aligned}
$$

Notice that, although derived from a scheme based on DPC (see the Appendix for the details), the rate region in (10) is achieved with only superposition coding since $T_{2}=\emptyset$ and $U_{2}$ is not precoded against $S_{1}$. By straightforward computations, the region in (10) can be shown to be at most 1.8 bits/user away from the upper bound in (9) when the condition

$$
\mathrm{S}_{\mathrm{c}} \frac{1+\mathrm{I}_{\mathrm{p}}+\mathrm{S}_{\mathrm{p}}}{1+2 \mathrm{I}_{\mathrm{p}}} \leq \mathrm{I}_{\mathrm{c}}
$$

holds - see Fig. 2 'Case C' on the right. We need to impose the condition in (11) to claim the redundancy of a bound of the form $R_{\mathrm{p}}+2 R_{\mathrm{c}}$ in the derived achievable region. Notice that the condition in (11) is similar to the condition in (6) derived to claim that bounds of the form $R_{\mathrm{p}}+2 R_{\mathrm{c}} / 2 R_{\mathrm{p}}+R_{\mathrm{c}}$ were redundant in the noncooperative achievable scheme.

D. The case $\mathrm{S}_{\mathrm{c}}>\mathrm{I}_{\mathrm{c}}$ and $\mathrm{C} \geq\left(\mathrm{S}_{\mathrm{p}}+\mathrm{I}_{\mathrm{c}}+2 \sqrt{\mathrm{S}_{\mathrm{p}} \mathrm{I}_{\mathrm{c}} \frac{\mathrm{I}_{\mathrm{p}}}{1+\mathrm{I}_{\mathrm{p}}}}\right)\left(1+\mathrm{I}_{\mathrm{p}}\right)$

In this regime, since $S_{c}>I_{c}$, we use (3) as outer bound on the capacity of the GCCIC. Here PTx takes advantage of the strong cooperation link and sends its message with the help of the CTx. The PTx has a (cooperative) private message only (carried by the pair $\left(S_{1}, Z_{1}\right)$ ), while the CTx 
has a (noncooperative) private message only (carried by $T_{2}$ ). In the DPC-based achievable scheme in the Appendix, the CTx, with knowledge of PTx's private message, uses DPC to rid CRx of the interference due to the primary private message. In a given time slot, CTx knows PTx's old private cooperative message and decodes PTx's new private cooperative message from its channel output. CTx then precodes its private noncooperative message against the 'known interference' $S_{1}$; thanks to DPC, CRx decodes its private message as if the interference $S_{1}$ was not present, while treating $Z_{1}$ as noise. PRx does backward decoding in order to recover its message while treating $T_{2}$ as noise. This is exactly the strategy described in the Appendix with $Q=V_{1}=U_{2}=\emptyset$, i.e., only a (cooperative) private message for PTx and a (noncooperative) private message for CTx. We apply Fourier-Motzkin elimination on the achievable region in (14) and we choose $Z_{1}, S_{1}, T_{2}^{\prime}$ to be i.i.d. $\mathcal{N}(0,1)$, and $X_{\mathrm{p}}=\left|\gamma_{\mathrm{p}}\right| \mathrm{e}^{\mathrm{j} \theta_{\mathrm{c}}} S_{1}+\sqrt{1-\left|\gamma_{\mathrm{p}}\right|^{2}} Z_{1}$, $X_{\mathrm{c}}=\left|\gamma_{\mathrm{c}}\right| S_{1}+\sqrt{1-\left|\gamma_{\mathrm{c}}\right|^{2}} T_{2}^{\prime}, T_{2}=T_{2}^{\prime}+\lambda S_{1}$ where the choice of $\lambda$ is so as to "pre-cancel" $S_{1}$ from $Y_{\mathrm{c}}$ in decoding $T_{2}$, i.e., so as to have $I\left(Y_{\mathrm{c}} ; T_{2}\right)-I\left(S_{1} ; T_{2}\right)=I\left(Y_{\mathrm{c}} ; T_{2} \mid S_{1}\right)$ [8]. Inspired by [13], we choose the power split $\gamma_{\mathrm{p}}$ in such a way that the interference created by $Z_{1}$ at $\mathrm{CRx}$ is at the same level of the noise, i.e, $\left|\gamma_{p}\right|^{2}=\frac{I_{p}}{1+I_{p}}$. With this choice of parameters the following rate region is achievable

$$
\begin{aligned}
& R_{\mathrm{p}} \leq \log \left(1+\frac{\mathrm{C}}{1+\mathrm{I}_{\mathrm{p}}}\right) \\
& R_{\mathrm{p}} \leq \log \left(1+\frac{\left.\mathrm{S}_{\mathrm{p}}+\left|\gamma_{\mathrm{c}}\right|^{2} \mathrm{I}_{\mathrm{c}}+2\left|\gamma_{\mathrm{c}}\right| \sqrt{\mathrm{I}_{\mathrm{c}} \mathrm{S}_{\mathrm{p}} \frac{\mathrm{I}_{\mathrm{p}}}{1+\mathrm{I}_{\mathrm{p}}}}\right)}{1+\left(1-\left|\gamma_{\mathrm{c}}\right|^{2}\right) \mathrm{I}_{\mathrm{c}}}\right) \\
& R_{\mathrm{c}} \leq\left(1+\frac{\left(1-\left|\gamma_{\mathrm{c}}\right|^{2}\right) \mathrm{S}_{\mathrm{c}}}{1+\frac{\mathrm{I}_{\mathrm{p}}}{1+\mathrm{I}_{\mathrm{p}}}}\right),
\end{aligned}
$$

for all $\left|\gamma_{c}\right| \leq 1$. With $C \geq\left(S_{p}+I_{c}+2 \sqrt{I_{c} S_{p} \frac{I_{p}}{1+I_{p}}}\right)\left(1+I_{p}\right)$ the constraint in (12a) is redundant and, straightforwardly, the region in (12) can be shown to be at most 1 bit/user away from the upper bound in (3).

Remark. If $S_{c}>I_{c}$, i.e., the PRx experiences weak interference, we cannot fix one "rate split" for the CTx $\left(\gamma_{c}\right)$ and claim optimality for all the rate region. Instead, we need to consider the union over all $\gamma_{c}$ (see (12)).

We have now concluded the proof of Th. 1. As can be noticed from the analysis above, the mixed and weak interference cases are more challenging than the strong interference case. In the journal version of this paper [12], we also studied two special cases of mixed / weak interference, namely the cases where one of the interfering links is absent. Due to the asymmetry in the cooperation, in [12] we considered both the case where CRx does not experience interference (i.e., the so-called Z-channel for which $\mathrm{I}_{\mathrm{p}}=0$ ) and the case where PRx does not experience interference (i.e., the so-called Schannel for which $\mathrm{I}_{\mathrm{c}}=0$ ). For both these channels, we characterized the capacity to within 2 bits/user. These results are not presented here for sake of space.

\section{CONCLUSIONS}

In this work we considered the CCIC, where, in contrast to the noncooperative IC, the CTx has access to primary information it receives over the air. This scenario represents a more practically relevant model for cognitive radio networks than the non-causal cognitive IC, where the CTx is assumed to have a priori knowledge of the PTx's message. Our major contribution was to design achievable schemes that match known outer bounds to within a constant gap for almost all parameters if, roughly speaking, the two destinations do not simultaneously suffer weak interference.

\section{APPENDIX A}

We specialize the 'binning+superposition' achievable scheme of [7, Sec. V]. In [7, Th. IV.1], the network comprises four nodes numbered from 1 to 4 ; nodes 1 and 2 are sources and nodes 3 and 4 destinations; source node $j \in[1: 2]$, with input to the channel $X_{j}$ and output from the channel $Y_{j}$, has a message $W_{j}$ for node $j+2$; destination node $j \in[3: 4]$ has channel output $Y_{j}$ from which it decodes the message $W_{j-2}$. Both users do rate splitting, where the messages of user 1 are cooperative while the messages of user 2 are noncooperative. In [7, Sec. V], we set $Y_{1}=U_{1}=T_{1}=S_{2}=V_{2}=Z_{2}=\emptyset$, i.e., then $R_{1}=R_{11 c}+R_{10 c}, R_{2}=R_{22 n}+R_{20 n}$. This scheme comprises: a cooperative common message (carried by the pair $\left(Q, V_{1}\right)$ at rate $\left.R_{10 c}\right)$ for user 1 , a cooperative private message (carried by the pair $\left(S_{1}, Z_{1}\right)$ at rate $R_{11 c}$ ) for user 1 , a noncooperative common message (carried by $U_{2}$ at rate $R_{20 n}$ ) for user 2 and a noncooperative private message (carried by $T_{2}$ at rate $\left.R_{22 n}\right)$ for user 2 . The pair $\left(Q, S_{1}\right)$ carries the 'past cooperative messages', and the pair $\left(V_{1}, Z_{1}\right)$ the 'new cooperative messages' in a block Markov encoding scheme. The channel inputs are functions of the auxiliary random variables, where $X_{1}$ is a function of $\left(Q, S_{1}, V_{1}, Z_{1}\right)$ and $X_{2}$ is a function of $\left(Q, S_{1}, U_{2}, T_{2}\right)$. The set of possible input distributions is

$$
\begin{array}{r}
P_{Q, S_{1}, V_{1}, Z_{1}, X_{1}, U_{2}, T_{2}, X_{2}}=P_{Q} P_{V_{1} \mid Q} P_{S_{1} \mid Q} P_{Z_{1} \mid Q, S_{1}, V_{1}} \\
P_{U_{2}, T_{2} \mid S_{1}, Q} P_{X_{1} \mid Q, S_{1}, V_{1}, Z_{1}} P_{X_{2} \mid Q, S_{1}, U_{2}, T_{2}}
\end{array}
$$

A schematic representation of the achievable scheme is given in Fig. 3, where a black arrow indicates superposition coding and a red arrow indicates binning / DPC [8].

Encoding. The codebooks are generated as follows: first the codebook $Q$ is generated; then the codebook $V_{1}$ is superposed to $Q$; independently of $V_{1}$, the codebook $S_{1}$ is superposed to $Q$ and then the codebook $Z_{1}$ is superposed to $\left(Q, S_{1}, V_{1}\right)$; independently of $\left(V_{1}, S_{1}, Z_{1}\right)$, the codebook $U_{2}$ is superposed to $Q$ and then the codebook $T_{2}$ is superposed to $\left(Q, U_{2}\right)$. With this random coding codebook generation, the pair $\left(U_{2}, T_{2}\right)$ is independent of $S_{1}$ conditioned on $Q$. [7, Th. V.1] involves several binning steps to allow for a large set of input distributions. Here, in order to simplify the scheme, we do not bin $V_{1}$ against $S_{1}$; the only binning steps are for $\left(U_{2}, T_{2}\right)$ against $S_{1}$. We use a block Markov coding scheme to convey the message of user 1 to user 2. In particular, at the end of any given 


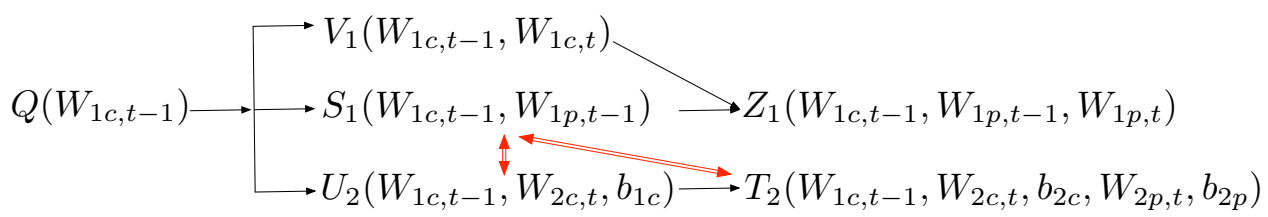

Fig. 3: Achievable scheme based on binning and superposition.

time slot in a block Markov coding scheme, encoder 2 knows $\left(Q, S_{1}, U_{2}, T_{2}\right)$ and decodes $\left(V_{1}, Z_{1}\right)$ from its channel output; the decoded pair $\left(V_{1}, Z_{1}\right)$ becomes the pair $\left(Q, S_{1}\right)$ in the next time slot; then, at the beginning of each time slot, encoder 2 , by binning, finds the new pair $\left(U_{2}, T_{2}\right)$ that is jointly typical with $\left(Q, S_{1}\right)$; for this to be possible, we must generate several $\left(U_{2}, T_{2}\right)$ sequences for each message of user 2 so as to be able to find one pair to send with the correct joint distribution with $\left(Q, S_{1}\right)$; this entails the rate penalties

$$
\begin{aligned}
& R_{20 n}^{\prime}+R_{22 n}^{\prime} \geq I\left(S_{1} ; U_{2}, T_{2} \mid Q\right), \\
& R_{20 n}^{\prime} \geq I\left(U_{2} ; S_{1} \mid Q\right) .
\end{aligned}
$$

Decoding. There are three decoding nodes in the network and therefore three groups of rate constraints.

- Node 2/CTx jointly decodes $\left(V_{1}, Z_{1}\right)$ from its channel output with knowledge of the indices in $\left(Q, S_{1}, U_{2}, T_{2}, X_{2}\right)$. Successful decoding is possible if

$$
\begin{aligned}
& R_{10 c}+R_{11 c} \leq I\left(Y_{2} ; Z_{1}, V_{1} \mid U_{2}, T_{2}, X_{2}, S_{1}, Q\right), \\
& R_{11 c} \leq I\left(Y_{2} ; Z_{1} \mid U_{2}, T_{2}, X_{2}, S_{1}, Q, V_{1}\right) .
\end{aligned}
$$

- Node 3/PRx jointly decodes $\left(Q, S_{1}, U_{2}\right)$ from its channel output, with knowledge of some message indices in $\left(V_{1}, Z_{1}\right)$, by treating $T_{2}$ as noise. Successful decoding is possible if

$$
\begin{aligned}
& R_{10 c}+R_{20 n}+ R_{11 c} \leq I\left(Y_{3} ; Q, V_{1}, S_{1}, Z_{1}, U_{2}\right) \\
&-\left(R_{20 n}^{\prime}-I\left(U_{2} ; S_{1} \mid Q\right)\right) \\
& R_{20 n}+R_{11 c} \leq I\left(Y_{3} ; S_{1}, Z_{1}, U_{2} \mid V_{1}, Q\right) \\
&-\left(R_{20 n}^{\prime}-I\left(U_{2} ; S_{1} \mid Q\right)\right) \\
& R_{11 c} \leq I\left(Y_{3} ; S_{1}, Z_{1} \mid V_{1}, Q, U_{2}\right)
\end{aligned}
$$

- Node $4 / \mathrm{CRx}$ jointly decodes $\left(Q, U_{2}, T_{2}\right)$ from its channel output, with knowledge of some message index in $V_{1}$, by treating $Z_{1}$ as noise (recall that the pair $\left(U_{2}, T_{2}\right)$ has been binned against $S_{1}$ ). Successful decoding is possible if

$$
\begin{aligned}
& R_{10 c}+R_{20 n}+R_{22 n} \leq I\left(Y_{4} ; U_{2}, T_{2}, V_{1}, Q\right) \\
& \quad-\left(R_{20 n}^{\prime}+R_{22 n}^{\prime}\right) \\
& R_{20 n}+R_{22 n} \leq I\left(Y_{4} ; U_{2}, T_{2} \mid V_{1}, Q\right)-\left(R_{20 n}^{\prime}+R_{22 n}^{\prime}\right) \\
& R_{22 n} \leq I\left(Y_{4} ; T_{2} \mid V_{1}, Q, U_{2}\right)-R_{22 n}^{\prime}
\end{aligned}
$$

For the GCCIC, we identify Node1 with the PTx (i.e., $X_{\mathrm{p}}=$ $X_{1}$ ), Node2 with the CTx (i.e., $X_{\mathrm{c}}=X_{2}, Y_{\mathrm{f}}=Y_{2}$ ), Node3 with the PRx (i.e., $Y_{\mathrm{p}}=Y_{3}$ ) and Node4 with the CRx (i.e., $Y_{\mathrm{c}}=Y_{4}$ ).

\section{ACKNOWLEDGMENT}

The work of Dr. D. Tuninetti was partially funded by NSF under award number 0643954. Eurecom's research is partially supported by its industrial partners: BMW Group Research \& Technology, IABG, Monaco Telecom, Orange, SAP, SFR, ST Microelectronics, Swisscom and Symantec. The research carried out at Eurecom leading to these results has received funding from the EU Celtic+ Framework Program Project SHARING. The research at IMC has received funding from the EU FP7 grant agreement iJOIN n 317941.

\section{REFERENCES}

[1] A. Goldsmith, S. A. Jafar, I. Maric, and S. Srinivasa, "Breaking spectrum gridlock with cognitive radios: An information theoretic perspective," Proocedings of the IEEE, vol. 97, no. 5, pp. 894 -914, May 2009.

[2] N. Devroye, P. Mitran, and V. Tarokh, "Achievable rates in cognitive radio channels," IEEE Trans. on Info. Theory, vol. 52, no. 5, pp. 1813 - 1827, May 2006.

[3] LTE-A, 3rd Generation Partnership Project; Technical Specification Group Radio Access Network; Evolved Universal Terrestrial Radio Access (EUTRA). 3GPP TR 36.806 V9.0.0, 2010.

[4] A. Host-Madsen, "Capacity bounds for cooperative diversity," IEEE Trans. on Info. Theory, vol. 52, no. 4, pp. 1522 -1544, April 2006.

[5] V. Prabhakaran and P. Viswanath, "Interference channels with source cooperation," IEEE Trans. on Info. Theory, vol. 57, no. 1, pp. 156 -186, January 2011.

[6] D. Tuninetti, "An outer bound region for interference channels with generalized feedback," in Information Theory and Applications Workshop (ITA), 2010, February 2010, pp. 1-5.

[7] S. Yang and D. Tuninetti, "Interference channel with generalized feedback (a.k.a. with source cooperation): Part i: Achievable region," IEEE Trans. on Info. Theory, vol. 57, no. 5, pp. 2686 -2710, May 2011.

[8] M. Costa, "Writing on dirty paper (corresp.)," IEEE Trans. on Info. Theory, vol. 29, no. 3, pp. 439 - 441, May 1983.

[9] S. Yang and D. Tuninetti, "Interference channels with source cooperation in the strong cooperation regime: Symmetric capacity to within 2 bits/s/hz with dirty paper coding," in Asilomar 2011, November 2011, pp. $2140-2144$.

[10] S. Rini, D. Tuninetti, and N. Devroye, "Inner and outer bounds for the Gaussian cognitive interference channel and new capacity results," IEEE Trans. on Info. Theory, vol. 58, no. 2, pp. 820 -848, February 2012.

[11] M. Mirmohseni, B. Akhbari, and M. Aref, "On the capacity of interference channel with causal and noncausal generalized feedback at the cognitive transmitter," IEEE Trans. on Info. Theory, vol. 58, no. 5, pp. 2813-2837, May 2012.

[12] M. Cardone, D. Tuninetti, R. Knopp, and U. Salim, "On the capacity of the two-user Gaussian causal cognitive interference channel," arXiv:1207.5319v3, September 2013.

[13] R. Etkin, D. Tse, and H. Wang, "Gaussian interference channel capacity to within one bit," IEEE Trans. on Info. Theory, vol. 54, no. 12, pp. $5534-5562$, December 2008

[14] M. Cardone, D. Tuninetti, R. Knopp, and U. Salim, "New outer bounds for the interference channel with unilateral source cooperation," submitted to IEEE International Symposium on Information Theory (ISIT), 2014, available at arXiv:1402.1572, 2014.

[15] C. Suh and D. Tse, "Feedback capacity of the Gaussian interference channel to within 2 bits," IEEE Trans. on Info. Theory, vol. 57, no. 5, pp. 2667-2685, May 2011. 